\title{
Fetal and Neonatal Cerebral Metabolism Following Maternal Canine Starvation
}

\author{
R. M. KLIEGMAN, ${ }^{(39)}$ E. L. MIETtineN, W. M. ROLIN, AND P. A. J. ADAM ${ }^{(37)}$ \\ Department of Pediatrics, Division of Pediatric Metabolism, Case Western Reserve University at Cleveland \\ Metropolitan General Hospital, and Rainbow Babies and Childrens Hospital, Cleveland, Ohio, USA
}

\begin{abstract}
Summary
Pregnant dogs were starved for $72 \mathrm{hr}$ before a term delivery. Maternal $(1.68 \pm 0.39$ versus $0.74 \pm 0.20 \mathrm{mM})$ and fetal $(0.39 \pm$ 0.03 versus $0.22 \pm 0.07$ ) circulating free fatty acids and maternal $(2.99 \pm 0.79$ versus $1.04 \pm 0.84)$ and fetal $(2.53 \pm 0.35$ versus 1.01 \pm 0.32 ) ketones were elevated whereas blood glucose values remained unchanged at the time of delivery. After birth, pups born to starved mothers had significantly lower blood glucose values during 3, 6, 9, and 24 hours of neonatal fasting. Intracerebral glucose concentrations paralleled those in the blood as they were depressed at 3, 6, and 9 hours of age. Cerebral glycogen content was lower in pups born to starved mothers at $6(2.72 \pm 0.43$ versus $4.32 \pm 0.56 \mu$ moles $/ g)$ and $24(2.31 \pm 0.17$ versus $3.48 \pm 0.39$ $\mu$ moles/g) hr, whereas UDP-glucose concentrations were significantly elevated in these pups during fetal, 3, 9, and $24 \mathrm{hr}$ of age. Phosphoenolpyruvate was higher after maternal starvation in the fetus and at 6 and $9 \mathrm{hr}$, whereas cerebral pyruvate concentrations were elevated at 3,6 , and $9 \mathrm{hr}$ of age. The elevation of pyruvate with no alteration of lactate concentration resulted in an elevated cytoplasmic NAD/NADH ratio at $3 \mathrm{hr}$ of age (1381 \pm 194 versus $792 \pm 198$ )

Cerebral $\alpha$-ketoglutarate and calculated oxaloacetate concentrations were elevated throughout the day after maternal starvation whereas malate concentrations were depressed at 3 and $9 \mathrm{hr}$ of age. Cerebral energy charge was unaffected, whereas the calculated energy reserve was lower at 3,6 , and 24 hours. Cerebral amino acids demonstrated elevated aspartate concentrations at 3 and $6 \mathrm{hr}$. Cerebral glutamine concentrations were lower during fetal stage (7.86 \pm 0.52 versus $10.01 \pm 0.41 \mu \mathrm{moles} / \mathrm{g})$ and 3,6 , and $9 \mathrm{hr}$ of life.
\end{abstract}

\section{Speculation}

These data suggest that cerebral glycogen synthesis was diminished and that the oxidation of cerebral amino acids, in particular glutamine, may contribute to maintain cerebral energy production during the relative fasting neonatal hypoglycemia which develops in pups after maternal starvations.

Nutritional deprivation during pregnancy may result in significant maternal and fetal hypoglycemia (2, 15, 17, 18). Fasting in pregnant humans and rats is characterized by an accelerated decline of maternal blood glucose concentrations and a reciprocal rapid elevation of ketone body levels (17). Consequently, the provision of glucose for fetal oxidative metabolism diminishes while the potential for fetal ketone body utilization increases ( 2 , $3)$. In addition, maternal ovine starvation significantly reduces fetal glucose consumption while acutely increasing the utilization of amino acids to support fetal oxidative metabolism (35).

Fetal hypoglycemia may have adverse effects on cerebral functional integrity. During insulin induced hypoglycemia, the ovine fetal electroencephalogram may demonstrate slowing and decreased voltage (27). This investigation also noted that fetal cere- bral oxygen consumption was not altered during hypoglycemia and that the cerebral uptake of alternate fuels was not augmented. These observations suggested that substrates endogenous to the fetal brain may have been oxidized to maintain cerebral energy production. Similar observations have been made during insulin induced hypoglycemia in adult rats (30). In addition, these investigations demonstrated a decline in murine cerebral glycolytic, tricarboxylic acid cycle, and amino acid intermediate pools (23, 30 ). These data further suggested that the utilization of endogenous cerebral substrates may serve as alternate fuels for energy production during periods of hypoglycemia.

Many of the models utilized to investigate hypoglycemia during fetal and neonatal life required the administration of insulin. Hyperinsulinism is not frequently observed during the fetal or neonatal hypoglycemia which follows maternal nutritional deprivation or intrauterine growth retardation. Insulin administration may also interfere with the systemic mobilization of alternate fuels because insulin inhibits lipolysis and subsequent hepatic ketogenesis (8). Presently there is little information pertaining to neonatal intracerebral metabolism during spontaneous hypoglycemia after maternal nutritional deprivation. This is a particularly important problem for intrauterine growth-retarded human newborns (11). During the investigation of the effects of maternal canine starvation on neonatal glucose homeostasis, significant fasting hypoglycemia developed (20). To evaluate the cerebral cortical metabolic events resulting from maternal canine starvation and subsequent neonatal hypoglycemia, we have determined cerebral glycolytic and tricarboxylic acid cycle intermediates as well as high-energy phosphate compounds. Because the "glutamate" group of cerebral amino acids is potentially a source of endogenous fuels, the relationship between these amino acids and their tricarboxylic acid cycle ketoacids was also investigated.

\section{MATERIALS AND METHODS}

\section{MATERIALS}

Alanine aminotransferase, amyloglucosidase (grade II), asparate aminotransferase (type I), citrate lyase, creatine phosphokinase (type I), glucose-6-phosphate dehydrogenase (type XV), glutaminase (grade V), hexokinase (type C-301), lactate dehydrogenase (type II), malic dehydrogenase, myokinase (grade III), phosphoglucose isomerase (type IV), pyruvate kinase (type II), and uridine5 -diphosphoglucose dehydrogenase (type III) were purchased from Sigma Chemical Co., St. Louis, MO. Glutamate dehydrogenase (A-grade) was purchased from CalBiochem, La Jolla, CA. All chemicals, cofactors, and standards were reagent grade.

\section{ANIMALS}

Pregnant beagles with known dates of confinement were housed in kennels and fed a standard diet. After delivery by cesarean section at term ( $60 \pm 2$ days), pups were fasted and housed in an Armstrong incubator in which the temperature was maintained at $37^{\circ} \mathrm{C}$ and humidity regulated at $70 \%$. 


\section{EXPERIMENTAL DESIGN}

\section{MOTHERS}

Before a term delivery, five dogs were given free access to water and starved for $72 \mathrm{hr}$, whereas five control mothers were fasted overnight. Before cesarean section, intravenous anesthesia was induced with $5 \mathrm{mg} / \mathrm{kg}$ of chlorpromazine while the abdominal incision site was infiltrated with $2 \%$ lidocaine.

\section{PUPS}

Fetal liver and brain from each litter were obtained immediately after delivery of the pup. Pups to be investigated later during the day were then delivered and removed from their amnionic membranes, stimulated, completely dried, and placed in a warmed incubator. All pups were healthy, viable, full-time, and acyanotic while breathing room air. Once stablized, pups had arterial and venous catheters (Argyle, St. Louis, MO) gently placed in their umbilical vessels and were then returned to the incubator. All pups rested quietly for the duration of the investigation.

After periods of neonatal fasting lasting 3, 6, 9, or $24 \mathrm{hr}$, a blood sample was withdrawn and replaced with an equivalent volume of isotonic saline. Then neonatal liver and cerebral cortex were rapidly isolated utilizing a double guillotine designed to simultaneously transect the abdomen and cranium. Immediately after being exposed (less than $3 \mathrm{sec}$ ), cerebral cortical tissue was quickly brought to the temperature of liquid nitrogen with previously cooled aluminum clamps. Tissue was subsequently stored at $-80^{\circ} \mathrm{C}$ until analyzed. This investigation concerns the analysis of cerebral cortical data.

\section{ANALYSES}

\section{CIRCULATING SUBSTRATES}

Blood samples were precipitated with $10 \%$ perchloric acid and neutralized with $\mathrm{K}_{2} \mathrm{CO}_{3}$. Glucose and lactate were enzymatically determined from the supernatant (1). Plasma acetoacetate and $\beta$ hydroxybutyrate were determined enzymatically (5), and free fatty acids were assayed by the colorimetric method of Novak (31) after extraction with Dole's reagent (13).

\section{INTRACEREBRAL SUBSTRATES AND INTERMEDIATES}

The frozen cerebral cortical tissues were weighed and pulverized to a fine powder at the temperature of dry ice, extracted, and homogenized with $3 \mathrm{M}$ perchloric acid in combination with 10 $\mathrm{mM}$ EDTA and then rapidly neutralized with $2 \mathrm{M} \mathrm{K}_{2} \mathrm{CO}_{3}$ at $0^{\circ} \mathrm{C}$. Cerebral glycogen was extracted and assayed enzymatically by the method of Vannucci and Duffy (36). Cerebral concentrations of uridinediphosphoglucose, glucose, glucose-6-phosphate, fructose6-phosphate, lactate, pyruvate, phosphoenolpyruvate (PEP), malate, citrate, and $\alpha$-ketoglutarate were determined fluorometrically following Lowry's methods (26) with minor modifications. Cerebral adenine nucleotide concentrations (ATP, ADP, and AMP) and creatine phosphate were assayed according to Lowry and Passonneau (26). Glutamine was determined according to Bergmeyer (5) and alanine, aspartate, and glutamate according to Lowry and Passonneau (26). Immediately after acid extraction and neutralization, aliquots were divided and stored at $-80^{\circ} \mathrm{C}$. ATP, ADP, AMP, and creatine phosphate were assayed as soon as possible, but never later than $1 \mathrm{wk}$ after neutralization. After these initial determinations, the remaining intermediates were performed. All analyses were assayed in duplicate.

\section{CALCULATIONS}

The cytoplasmic NAD/NADH ratio was calculated from the equation (12):

$$
[\mathrm{NAD}] /[\mathrm{NADH}]=[\text { pyruvate }] /\left(\mathrm{K}_{\mathrm{LDH}}\right)[\text { lactate }]
$$

where $\mathrm{K}_{\mathrm{LDH}}=111 \times 10^{-6}$ at $\mathrm{pH} 7.0$. Oxaloacetate was calculated from the equation (12):

$$
\text { Oxaloacetate } \left.=[\alpha \text {-ketoglutarate }] \text { [aspartate }] /\left(\mathrm{K}_{\mathrm{GOT}}\right) \text { [glutamate }\right]
$$

where $\mathrm{K}_{\mathrm{GOT}}=6.61$ at $\mathrm{pH} 7.0$. Cerebral energy charge was calculated from the equation (4):

$$
\text { Energy charge }=(\mathrm{ATP}+0.5 \mathrm{ADP}) /(\mathrm{ATP}+\mathrm{ADP}+\mathrm{AMP})
$$

Cerebral energy reserve was calculated as follows (12):

$$
\begin{aligned}
\text { Energy reserve }= & (\text { creatine phosphate }+ \text { ADP })+2(\text { ATP }+ \\
& \text { glucose })+2.9 \text { (glycogen }) \\
& \text { STATISTICAL ANALYSES }
\end{aligned}
$$

All notations are recorded as the mean \pm standard error of the mean (S.E.). Concentrations are expressed as $\mu$ moles/g tissue wet weight. Standard calculator programs were used for Student's $t$ test analysis of data between the groups.

\section{RESULTS}

\section{CIRCULATING FUELS (TABLEI)}

As noted in Table 1, $72 \mathrm{hr}$ of maternal canine starvation had no effect on maternal or fetal blood glucose concentrations at the time of delivery. However, after birth, the blood glucose concentration in pups of starved mothers was significantly depressed after $3,6,9$, or $24 \mathrm{hr}$ of neonatal fasting. Despite this relative hypoglycemia, both groups of pups had similar neurological behavior and activity. Maternal starvation had no effect on maternal, fetal, or fasting neonatal blood lactate concentrations; however, there were significant elevations of both maternal and fetal plasma ketone bodies and free fatty acids. After birth, neonatal total ketone body concentrations declined and became equivalent in both pup groups; however, plasma free fatty acid levels were depressed in pups of starved mothers after $3 \mathrm{hr}$ of neonatal fasting. During the remainder of the study, free fatty acids achieved control values and were not significantly different throughout the day.

\section{INTRACEREBRAL METABOLITES}

Cerebral Glycolytic Intermediates (Figs. 1 and 2). Fetal cerebral glycogen content was not affected by $72 \mathrm{hr}$ of maternal starvation. After birth, cerebral glycogen values increased in control pups and achieved maximum concentrations at $6 \mathrm{hr}$ of age. Pups subjected to prior maternal starvation had an initial elevation of cerebral glycogen content at $3 \mathrm{hr}$ of age; however, by 6 and $24 \mathrm{hr}$, glycogen levels were now significantly lower than in control pups. Intracerebral UDP-glucose levels were stable in control pups from fetal to $6 \mathrm{hr}$ of life and then declined at 9 and $24 \mathrm{hr}$. In contrast, after maternal starvation, cerebral UDP-glucose concentrations were elevated throughout the first day, being significant during fetal life and after 3, 9, and $24 \mathrm{hr}$ of neonatal fasting.

Fetal cerebral glucose as well as blood glucose concentrations were unaffected by prior maternal starvation. After birth, cerebral glucose values in control pups rose, paralleling the rise in blood glucose, whereas the cerebral glucose concentrations in pups of starved mothers became significantly depressed at $3 \mathrm{hr}$ of age. Cerebral glucose values remained significantly lower than in controls from this time until $9 \mathrm{hr}$ of age; nonetheless, by $24 \mathrm{hr}$ of age cerebral glucose levels were equivalent in both groups. To determine the relationship of the cerebral glucose to circulating glucose concentration, the cerebral glucose to blood glucose ratio was calculated. This ratio may also be used as an indicator of intracerebral glucose utilization. During the day, the ratio remained the same in control pups, whereas in pups of starved mothers, it was significantly elevated after 3 and $24 \mathrm{hr}$ of neonatal fasting.

Fetal and neonatal cerebral glucose-6-phosphate concentrations were stable throughout the day and were unaffected by prior 
Table 1. Effect of maternal starvation on blood and plasma levels of fuels in canine mother, fetus, and newborn ( $\mu m o l e s / m l)$

\begin{tabular}{|c|c|c|c|c|c|c|}
\hline & \multicolumn{2}{|c|}{ At birth } & \multicolumn{4}{|c|}{ Newborn dog (hr) } \\
\hline & Mother & Fetus & 3 & 6 & 9 & 24 \\
\hline \multicolumn{7}{|l|}{$\mathrm{B}^{1}$-Glucose } \\
\hline Control & $3.75 \pm 0.87^{2}$ & $3.71 \pm 1.20$ & $8.31 \pm 0.86$ & $9.23 \pm 1.39$ & $8.74 \pm 0.85$ & $8.23 \pm 0.89$ \\
\hline MCS & $2.85 \pm 0.72$ & $2.80 \pm 0.61$ & $2.50 \pm 0.46^{3}$ & $4.00 \pm 0.69^{4}$ & $2.94 \pm 0.30^{3}$ & $5.50 \pm 0.77^{5}$ \\
\hline \multicolumn{7}{|l|}{ B-Lactate } \\
\hline Control & $1.50 \pm 0.31$ & $3.50 \pm 0.49$ & $1.82 \pm 0.21$ & $2.09 \pm 0.28$ & $1.63 \pm 0.16$ & $2.33 \pm 0.26$ \\
\hline MCS & $1.45 \pm 0.26$ & $3.86 \pm 0.56$ & $1.70 \pm 0.27$ & $1.86 \pm 0.33$ & $1.44 \pm 0.09$ & $1.83 \pm 0.39$ \\
\hline \multicolumn{7}{|l|}{ P-Ketones } \\
\hline Control & $1.04 \pm 0.84$ & $1.01 \pm 0.32$ & $0.34 \pm 0.06$ & $0.39 \pm 0.05$ & $0.39 \pm 0.06$ & $0.37 \pm 0.07$ \\
\hline MCS & $2.99 \pm 0.79^{5}$ & $2.53 \pm 0.35^{4}$ & $0.27 \pm 0.02$ & $0.32 \pm 0.03$ & $0.30 \pm 0.08$ & $0.51 \pm 0.08$ \\
\hline \multicolumn{7}{|l|}{ P-FFA } \\
\hline Control & $0.74 \pm 0.20$ & $0.22 \pm 0.07$ & $1.01 \pm 0.09$ & $0.94 \pm 0.08$ & $0.88 \pm 0.10$ & $0.95 \pm 0.21$ \\
\hline MCS & $1.68 \pm 0.39^{5}$ & $0.39 \pm 0.03^{5}$ & $0.78 \pm 0.05^{5}$ & $1.01 \pm 0.15$ & $1.00 \pm 0.05$ & $0.55 \pm 0.07$ \\
\hline \multicolumn{7}{|l|}{ No. studied } \\
\hline Control & 5 & 5 & 7 & 8 & 7 & 6 \\
\hline MCS & 5 & 5 & 8 & 8 & 5 & 6 \\
\hline
\end{tabular}

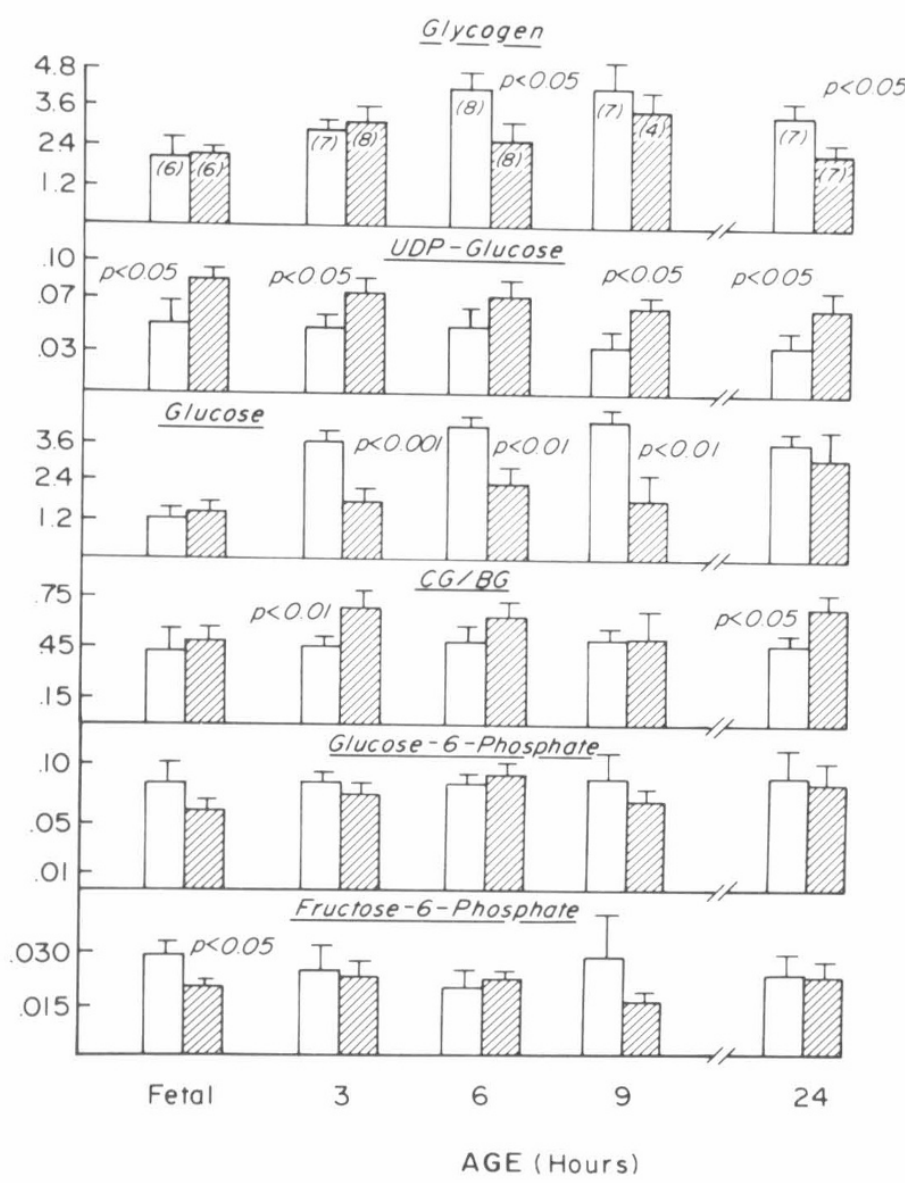

Fig. 1. Effect of maternal canine starvation on fetal and neonatal cerebral glycogen, UDP-glucose, glucose, glucose-6-phosphate, and fructose-6-phosphate concentrations ( $\mu$ moles/g) and cerebral-to-blood glucose $(C G / B G)$ ratio (mean \pm S.E.). $\square$, control pups; tion. Numbers in parentheses, number of pups.

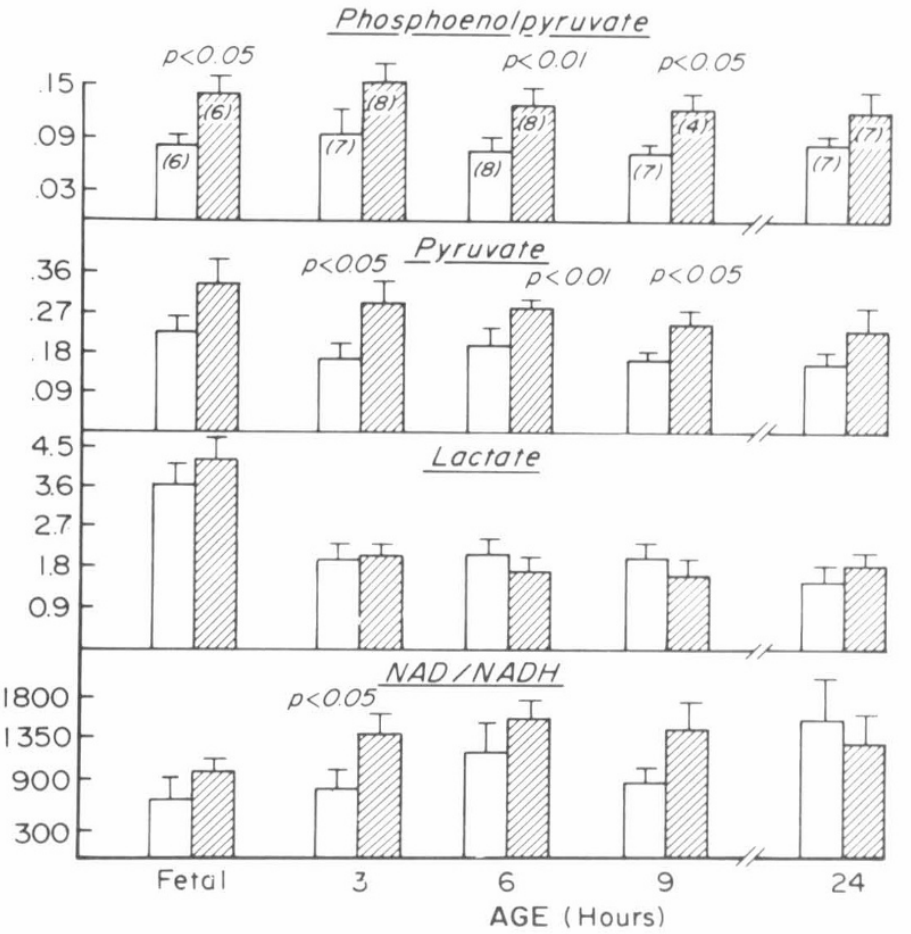

Fig. 2. Fetal and neonatal cerebral PEP, pyruvate, and lactate concentrations $(\mu$ moles $/ \mathrm{g})$ and calculated cytoplasmic NAD/NADH ratio (mean \pm S.E.). $\square$, control pups; prior maternal starvation. Numbers in parentheses, number of pups.

maternal starvation. Cerebral fructose-6-phosphate was signifcantly lower in the fetus after maternal starvation, but thereafter neonatal values were equivalent during the day.

As noted in Figure 2, intracerebral concentrations of PEP were significantly elevated after maternal starvation during fetal life and at 6 and $9 \mathrm{hr}$ of age. Although fetal cerebral pyruvate values 
Table 2. Cerebral amino acid concentrations ( $\mu$ moles/g)

\begin{tabular}{|c|c|c|c|c|c|}
\hline & Fetal & $3 \mathrm{hr}$ & $6 \mathrm{hr}$ & $9 \mathrm{hr}$ & $24 \mathrm{hr}$ \\
\hline \multicolumn{6}{|c|}{ Alanine } \\
\hline $\mathrm{C}^{1}$ & $0.504 \pm 0.094^{2}$ & $0.333 \pm 0.031$ & $0.318 \pm 0.044$ & $0.322 \pm 0.033$ & $0.331 \pm 0.0$ \\
\hline $\mathrm{S}$ & $0.739 \pm 0.085$ & $0.365 \pm 0.039$ & $0.424 \pm 0.081$ & $0.361 \pm 0.041$ & $0.379 \pm 0.0$ \\
\hline \multicolumn{6}{|c|}{ Aspartate } \\
\hline C & $0.762 \pm 0.046$ & $0.657 \pm 0.064$ & $0.597 \pm 0.060$ & $0.639 \pm 0.054$ & $0.672 \pm 0.0$ \\
\hline $\mathrm{S}$ & $0.907 \pm 0.120$ & $0.894 \pm 0.067^{3}$ & $0.821 \pm 0.053^{3}$ & $0.773 \pm 0.101$ & $0.775 \pm 0.0$ \\
\hline \multicolumn{6}{|c|}{ Glutamate } \\
\hline C & $6.27 \pm 0.83$ & $4.47 \pm 0.33$ & $3.74 \pm 0.32$ & $3.56 \pm 0.30$ & $4.33 \pm 0.3$ \\
\hline $\mathrm{S}$ & $5.57 \pm 0.39$ & $3.86 \pm 0.25$ & $4.29 \pm 0.41$ & $4.46 \pm 0.15$ & $4.56 \pm 0.4$ \\
\hline \multicolumn{6}{|c|}{ Glutamine } \\
\hline $\mathrm{C}$ & $10.01 \pm 0.41$ & $9.26 \pm 0.32$ & $8.45 \pm 0.33$ & $8.02 \pm 0.46$ & $8.46 \pm 0.7$ \\
\hline $\mathrm{S}$ & $7.86 \pm 0.52^{4}$ & $7.12 \pm 0.55^{4}$ & $6.85 \pm 0.43^{4}$ & $6.91 \pm 0.41^{4}$ & $7.26 \pm 0.6$ \\
\hline
\end{tabular}

were not significantly elevated, after birth, intracerebral pyruvate concentrations became significantly higher at 3,6, and $9 \mathrm{hr}$ of age in pups subjected to prior maternal starvation. As expected, cerebral lactate levels were higher in the fetus than the neonate. Prior maternal starvation and the various postnatal periods of fasting produced no differences in cerebral lactate levels among the pups.

The calculated cytoplasmic NAD/NADH ratio is also recorded in Figure 2. During the fetal period and at 3, 6, and $9 \mathrm{hr}$ of neonatal life, this redox ratio tended to be more oxidized in pups of starved mothers; however, this reached statistical significance only at $3 \mathrm{hr}$ of age.

Cerebral Tricarboxylic Acid Cycle Intermediates (Fig. 3). As seen in Figure 3, fetal and neonatal intracerebral citrate levels were stable during the first neonatal day and unaffected by prior maternal fasting. Control pups maintained constant $\alpha$-ketoglutarate levels during fetal and neonatal life. In contrast, $\alpha$-ketoglutarate concentrations were significantly elevated among pups of starved mothers, both during fetal life and all periods of neonatal fasting.

The malate-oxaloacetate equilibrium apparently reflects the relationship in redox state noted above. Fetal malate concentrations were not changed during maternal starvation; however, after birth and neonatal fasting for 3 and $9 \mathrm{hr}$, intracerebral malate values were significantly depressed. In contrast, reciprocal changes were noted across the malate-oxaloacetate equilibrium because the calculated oxaloacetate concentrations were significantly elevated in pups from starved mothers during both fetal and neonatal life.

Cerebral Amino Acids (Table 2). Because pyruvate, oxaloacetate, and $\alpha$-ketoglutarate concentrations were elevated at various periods after maternal starvation, the amino acids which may contribute to these intermediates by transamination reactions were determined. In control dogs after birth, intracerebral alanine concentrations declined at $3 \mathrm{hr}$ of age and remained stable throughout the day. Cerebral alanine levels were not affected by prior maternal starvation and demonstrated a similar postnatal pattern as that in control pups. Cerebral aspartate concentrations appeared to be elevated during fetal and neonatal life in pups of starved mothers, but this difference reached statistical significance only at 3 and $6 \mathrm{hr}$ of age.

Among control pups, cerebral glutamate concentrations declined after birth and thereafter stabilized during the period of neonatal fasting. As with alanine, prior maternal starvation had no affect on glutamate levels nor on the equivalent postnatal decline of this amino acid's intracellular concentration. Glutamine concentrations declined after birth in control pups, reaching a steady state level by $6 \mathrm{hr}$ of age. After maternal starvation,

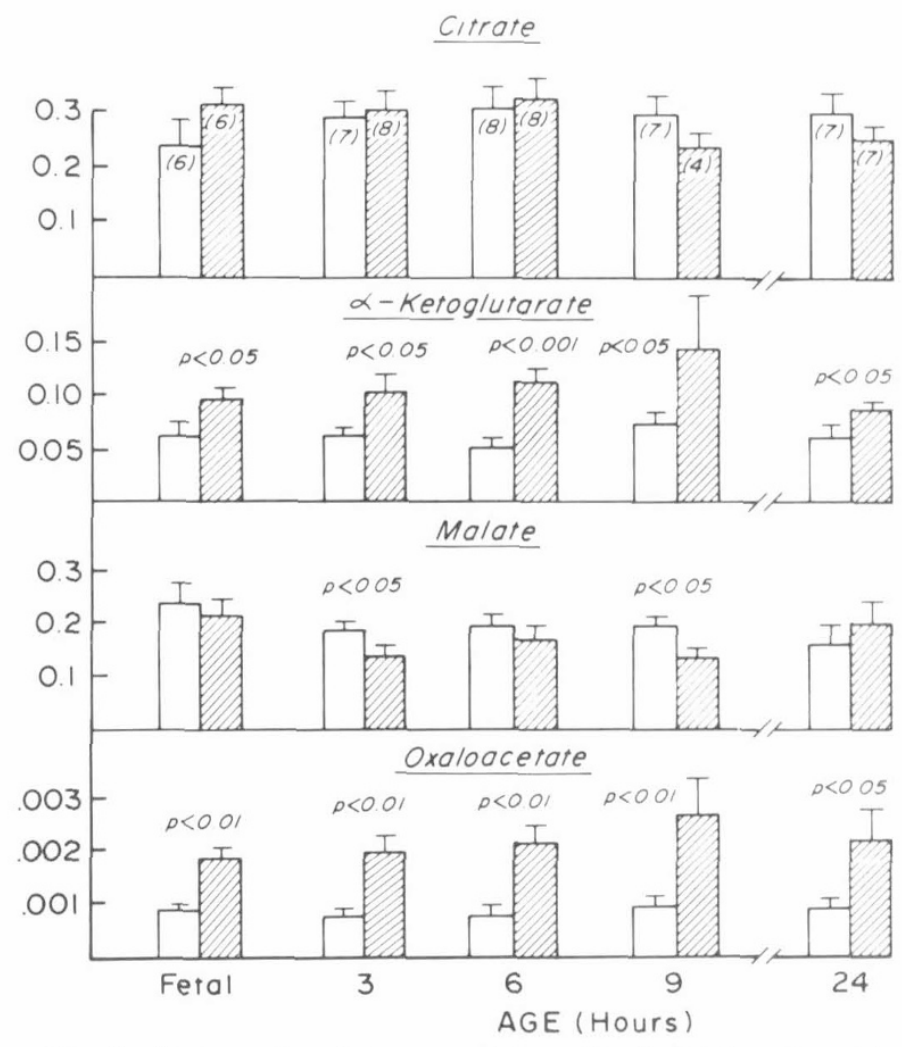

Fig. 3. Citrate, $\alpha$-ketoglutarate, malate, and calculated oxaloacetate concentrations ( $\mu$ moles $/$ g); mean \pm S.E. $\square$, control pups; prior maternal starvation. Numbers in parentheses, number of pups.

however, fetal cerebral glutamine concentrations started significantly lower at birth and continued to be depressed at 3,6, and 9 $\mathrm{hr}$ of neonatal life.

High-Energy Phosphate Compounds (Fig. 4). As reported in Figure 4, prior maternal starvation and postnatal adaptation had no effect on cerebral creatine phosphate, ATP, or ADP concentrations. AMP concentrations declined during the day in control pups, whereas levels among pups of starved mothers remained unchanged. The calculated cerebral energy charge also was unaffected during the postnatal adaptive periods of fasting or by maternal starvation. Nonetheless, the postnatal increase of the calculated energy reserve was affected in pups of starved mothers. In control pups the energy reserve increased after birth and 


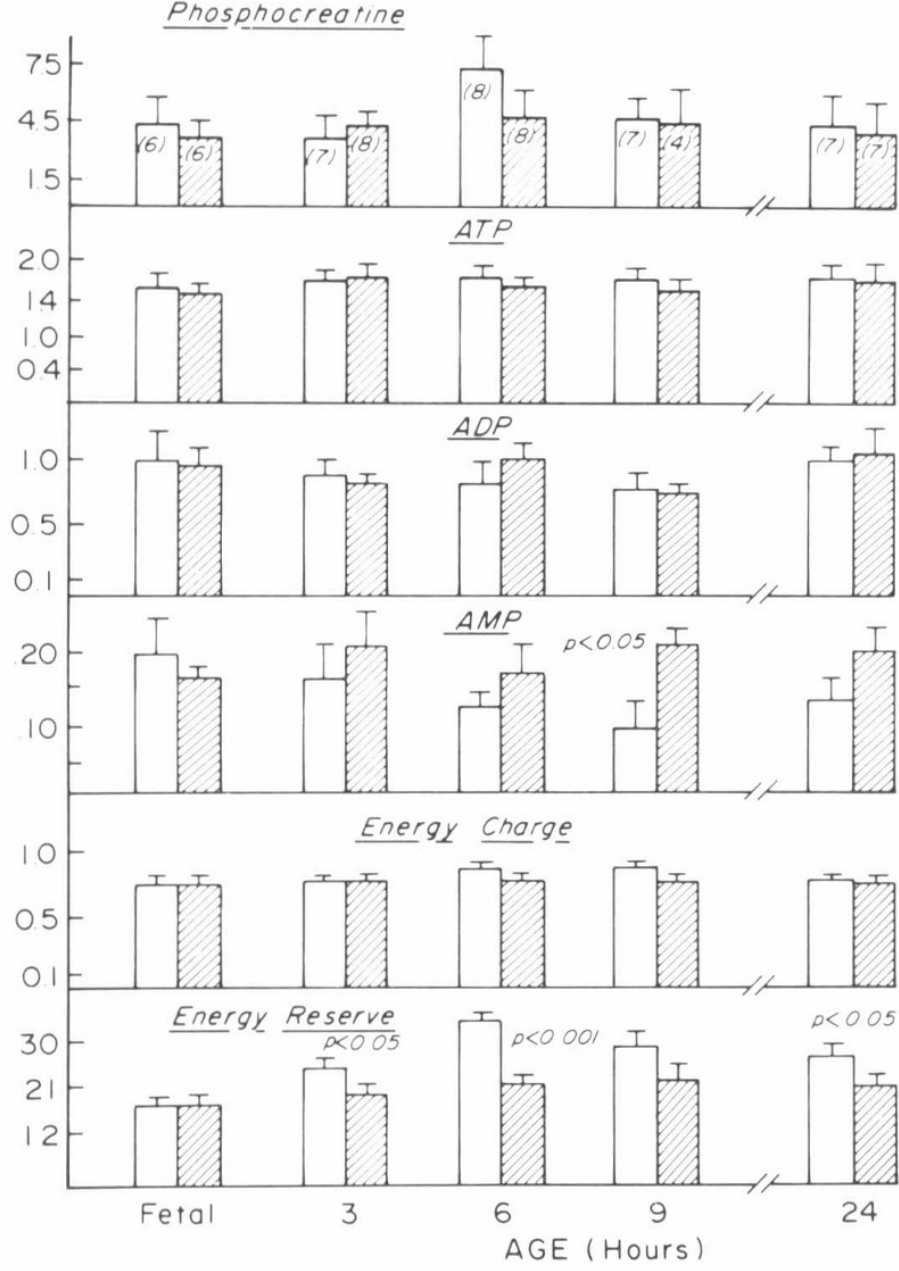

Fig. 4. Cerebral high-energy phosphate compounds $(\mu \mathrm{moles} / \mathrm{g})$ and calculated energy charge and reserve (mean \pm S.E.). $\square$, control pups; $\mathbb{2}$, prior maternal starvation. Numbers in parentheses, number of pups.

maintained this value throughout the day. In contrast, subsequent to maternal starvation, there was no elevation after birth because the calculated energy reserve was lower during 3,6 , and $24 \mathrm{hr}$ of neonatal fasting.

\section{DISCUSSION}

\section{CIRCULATING FUELS}

During mammalian fasting, alternate fuels such as free fatty acids and ketones are mobilized and provide as substrates to support oxidative metabolism (29). Because free fatty acids are not taken up by ovine fetal (27), human neonatal (22), or adult brain (32), this alternate fuel cannot directly affect cerebral metabolism during periods of starvation. Ketones, however, may be taken up and utilized by the fetal (3), neonatal (22), and adult human central nervous system (32). Despite this utilization of ketone bodies, the central nervous system requires a continuous provision of glucose as a substrate for energy production, thereby obligating a continuous, albeit diminished, hepatic glucose production. To spare glucose for the central nervous system, peripheral glucose utilization is reduced by the oxidation of free fatty acids or ketones (29).

Once born, pups of starved mothers developed significantly lower fasting blood glucose concentrations at 3, 6, 9, and $24 \mathrm{hr}$ of age. Similar observations have been reported for human infants after intrauterine growth retardation due in some circumstances to maternal nutritional deprivation (11). As expected, starvation produced elevated maternal and fetal levels of free fatty acids and ketone bodies. After birth, total ketone body concentrations de- clined and were equivalent in both groups, whereas pups from starved mothers demonstrated attenuated plasma levels of free fatty acids at $3 \mathrm{hr}$ of age. Intrauterine growth-retarded human neonates also have demonstrated a relative attenuation of free fatty acid levels after birth (11). In the present model, within $3 \mathrm{hr}$ of birth, pups developed significantly lower blood glucose and plasma free fatty acid concentrations. These metabolic changes have occurred spontaneously as part of the postnatal response to neonatal fasting in pups subjected to maternal starvation. Plasma insulin concentrations were appropriately suppressed during the periods of the lower blood glucose values (Kliegman, R. M., Miettinen, E. L., and Adam, P. A. J., unpublished results). Therefore, the perturbations produced in previous investigations after the administration of pharmacologic doses of insulin should not complicate the interpretation of the metabolic changes related to hypoglycemia and the neonatal central nervous system.

\section{CEREBRAL GLUCOSE METABOLISM}

Cerebral glucose concentrations paralleled the postnatal changes of blood glucose in both control and experimental pups. Because experimental pups experienced lower blood glucose concentrations, this resulted in a significant depression of cerebral glucose levels from 3 to $9 \mathrm{hr}$ after birth in pups of starved mothers. Although the fetal cerebral to blood glucose ratio was identical in both groups, this ratio became significantly elevated in pups of starved mothers at two time periods during neonatal fasting. Because the reduced provision of substrate for cerebral glycolysis may have limited flux through this pathway, such an altered ratio may have reflected diminished cortical glucose utilization (28). Such a supposition would have to assume that glucose transport across the neonatal canine blood brain barrier was not rate limiting in the range of blood glucose concentrations observed. Similarly, one would have to assume that transport into the brain was not enhanced at low cerebral or blood glucose concentrations. Collaborative data for diminished glycolytic flux has been reported during insulin induced hypoglycemia in the ovine fetus (27) and murine and human adults (34), in that cerebral glucose utilization declined as the blood glucose concentration was lowered. This supports the suggestion that cerebral glycolytic flux was possibly diminished in pups subjected to prior maternal starvation.

After birth, the newborn dog increased its cerebral glycogen content. Similar observations have been noted after birth in newborn rats (21). Subsequent to maternal starvation, newborn dogs demonstrated a transient increase of glycogen at $3 \mathrm{hr}$; however, at 6 and $24 \mathrm{hr}$ cerebral glycogen content became significantly lower than that of controls. This suggests a temporary increase of net cerebral glycogen synthesis followed by longer periods of diminished synthesis and/or enhanced glycogenolysis in newborn pups after maternal starvation. Other investigations have noted diminished cerebral glycogen content after insulin induced neonatal hypoglycemia in rats (9) and kittens (16). Of particular interest in the present investigation was the elevated levels of cerebral UDP-glucose noted during fetal and most of neonatal life in pups of starved mothers. As UDP-glucose concentrations declined during periods of active glycogen synthesis in the rat liver (14) and both neonatal and adult rat brain $(7,21)$, these data suggest that net glycogen synthetic activity was diminished in the fetus and pups of starved mothers.

\section{CEREBRAL AMINO ACID AND RELATED INTERMEDIATES}

At various time periods, cerebral pyruvate, $\alpha$-ketoglutarate, and calculated oxaloacetate concentrations were elevated in pups of starved mothers. Each of these intermediates partakes in transamination reactions which are capable of occurring in neonatal brain. These reactions are able to draw upon the large pool of cerebral amino acids, thus providing additional endogenous substrates for cerebral oxidative metabolism. During insulin-induced hypoglycemia in adult rats, cerebral oxygen consumption usually remains unchanged or declines minimally compared to the markedly reduced rate of cerebral glucose utilization (30). Under these 
conditions, additional circulating alternate substrates are not taken up by the brain, thereby necessitating the oxidation of endogenous substrates to maintain ongoing cerebral energy production $(23,24$, $30)$.

In pups of starved mothers, cerebral pyruvate concentrations were significantly elevated at 3, 6, and 9 hr of life, whereas lactate levels were unaffected. The calculated elevation of the cytoplasmic NAD/NADH redox state at $3 \mathrm{hr}$ of age suggest that the cytoplasm was more oxidized in these pups. This redox relationship may also be reflected in the malate-oxaloacetate equilibrium. In pups of starved mothers, measured cerebral malate concentrations were depressed at 3 and $9 \mathrm{hr}$, whereas calculated oxaloacetate values were elevated throughout the study period. The oxidized nature of the cytoplasm in these pups suggests diminished flux through the cytoplasmic energy producing pathways which also generate NADH (34). As suggested above, this may be due to diminished glycolytic flux during periods of low cerebral glucose concentrations in pups of starved mothers.

Cerebral glutamine concentrations were significantly depressed during fetal and after 3,6, and $9 \mathrm{hr}$ of fasting in pups of starved mothers. After conversion to glutamate, glutamine carbons may enter the tricarboxylic acid cycle by way of transamination or by oxidative deamination of glutamate to $\alpha$-ketoglutarate and contribute to net cerebral energy production. The diminished glutamine concentrations and simultaneously enhanced $\alpha$-ketoglutarate values suggest increased flux through these pathways. Similarly, once glutamine is converted to glutamate, the transamination reaction:

\section{Glutamate + oxaloacetate $=$ aspartate $+\alpha$-ketoglutarate}

may result in an elevation of cerebral aspartate levels (34). An elevation of cerebral aspartate levels was observed in pups of starved mothers at 3 and $6 \mathrm{hr}$ of age. Insulin-induced hypoglycemia in neonatal (10) or adult rats (23) produced similar reciprocal changes in cerebral glutamine and aspartate concentrations. In addition, glutamate concentrations also declined, but this was not constant in each age group studied (10).

Once glutamine carbons enter the tricarboxylic acid cycle, they may be oxidized and converted to oxaloacetic acid. At this point if no further reaction occurs, oxaloacetic acid may accumulate, and continued energy production would cease. As glycolytic flux may be diminished, a deficiency of acetyl-CoA to combine with oxaloacetic acid for the formation of citrate may occur. This relative deficiency of acetyl-coenzyme A (acetyl-CoA) may also result in elevated oxaloacetate levels. Intracellular citrate concentrations were equivalent in both groups, suggesting that there was an additional pathway providing carbons for acetyl-CoA production. Cerebral ketone oxidation may provide carbons for acetylCoA production (3). However, the contribution of ketones to cerebral oxidative metabolism was probably minimal, as their plasma concentrations remained low during the period of neonatal fasting. One potential reaction is for oxaloacetic acid to be converted to PEP by way of pyruvate carboxylase (34). Subsequent conversion of PEP to pyruvate and pyruvate to acetyl-CoA, may enable the complete oxidation of these carbons derived originally from endogenous amino acid precursor substrates (34). Aside from the altered NAD/NADH ratio shifting the lactate-pyruvate equilibrium toward pyruvate, the elevation of cerebral pyruvate and PEP concentrations may reflect increased flux through the previously discussed alternate pathways involving these intermediates. An additional contributing factor explaining the elevation of PEP may also include the possibility that enzymatic regulation was operating on pyruvate kinase, which potentially may be one ratelimiting glycolytic enzyme in cerebral $(25,33)$ and other $(6)$ tissues.

\section{CEREBRAL ENERGY METABOLISM}

Prior experiments with insulin-induced hypoglycemia in neonatal (9) and adult rats (24) have demonstrated preservation of cerebral oxygen consumption, ATP, and creatine phosphate levels during periods of moderate hypoglycemia (24). With severe hy- poglycemia (cerebral glucose $<1 \mu$ mole/g), ATP and creatine phosphate levels declined, suggesting an imbalance between energy production and utilization. Nevertheless, before this state, cerebral levels of high-energy phosphate compounds were preserved $(19,24)$.

During the periods of hypoglycemia, the pups of starved mothers maintained normal control levels of cerebral ATP and creatine phosphate. Similarly, prior maternal starvation and the subsequent fasting neonatal hypoglycemia had no effect on the cerebral energy charge. If cerebral cortical glycolytic flux had diminished in these pups, as reflected by decreased cytoplasmic NADH generation and altered cerebral to blood glucose ratio, an alternate source for energy production must have been provided to maintain the energy charge. Assuming no alteration of cerebral oxygen consumption, then alternate fuels need to be oxidized within the mitochondria by way of the tricarboxylic acid cycle. The changes in cerebral glutamine, aspartate, oxaloacetate, and $\alpha$-ketoglutarate suggest that the relatively large pool of cerebral amino acids, in particular glutamine, were being oxidized to support cerebral energy requirements $(10,19,23,30)$.

The calculated cerebral energy reserve, however, was not similar to control values at 3,6 , and $24 \mathrm{hr}$ of age. This may reflect diminished net glycogen synthesis with ongoing glycogen consumption, but also may result from lower cerebral glucose concentrations. Nevertheless, despite the ability of the neonatal canine brain to maintain the energy charge during hypoglycemia, the net synthesis of glycogen depots appears to be inhibited relative to control pups.

\section{CONCLUSION}

Maternal canine starvation for $72 \mathrm{hr}$ before birth results in significantly lower neonatal blood glucose concentrations during fasting for 3 to $24 \mathrm{hr}$. Neonatal cerebral glycogen synthesis at times may be inhibited whereas cerebral amino acids, specifically glutamine, may enter the mitrochondrial oxidative pathway to maintain the energy state of the brain. Although energy production seems unaffected and cerebral function appeared normal, it is possible that this is at the expense of synthetic processes, exemplified by diminished postnatal glycogen deposition.

\section{REFERENCES AND NOTES}

1. Adam, P. A. J., Glazer, G., and Rogoff, F.: Glucose production in the newborn dog. I. Effects of glucagon in vivo. Pediatr. Res., 9: 816 (1975).

2. Adam, P. A. J., and Felig, P.: Carbohydrate, fat and amino acid metabolism in the pregnant woman and fetus. In: F. Falkner, J. M. Tanner: Human Growth. Vol. 1, p. 461 (Plenum Publishing Corporation, 1978).

3. Adam, P. A. J., Räihä, N., Rahiala. E-L., and M. Kekomaki: Oxidation of glucose and $\mathrm{D}-\beta-\mathrm{OH}$-butyrate by the early human fetal brain. Acta Pediatr. Scand. 64 17 (1975).

4. Atkinson, D. E. and Walton, G. M.: Adenosine triphosphate conservation in metabolic regulation. J. Biol. Chem., 242: 3239 (1967).

5. Bergmeyer, H.: Methods of Enzymatic Analysis. Ed. 2 (Academic Press, Inc., New York, 1974.)

6. Blair, J. B., Cimbala, M. A., Foster, J. L., and Morgan. R. A.: Heptatic pyruvate kinase. J. Biol. Chem.. 251: 3756 (1976).

7. Brunner, E. A., Passonneau, J. V., and Molstad, C.: The effect of volatile anaesthetics on levels of metabolites and on metabolic rate in brain. J. Neurochem., 18: 2301 (1971)

8. Cahill, G. F., Jr.: Physiology of insulin in man. Diabetes, 20: 785 (1971).

9. Chase, H. P., Marlow, R. A., Dabiere, C. S., and Welch N. N.: Hypoglycemia and brain development. Pediatrics, 52: 513 (1973).

10. Davis, J. M., Himwich, W. A., and Pederson, V. C.: Hypoglycemia and developmental changes in free amino acids of rat brain. J. Appl. Physiol., 29:219 (1970).

11. DeLeeuw, R. and DeVris, I.: Hypoglycemia in small for dates newborn infants. Pediatrics, 58: 18 (1976).

12. DeVivo, D. C., Leckie, M. P., Ferrendelli, J. S., and Mc Dougal, D. B.: Chronic ketosis and cerebral metabolism. Ann. Neurol., 3: 331 (1978).

13. Dole, V. P.: A relation between non-esterified fatty acids in plasma and the metabolism of glucose. J. Clin. Invest., 35: 150 (1956).

14. El-Refai, M., and Bergman, R. N.: Simulation study of control of hepatic glycogen synthesis by glucose and insulin. Am. J. Physiol., 231: 1608 (1976).

15. Felig. P.: Maternal and fetal fuel homeostasis in human pregnancy. Am. J. Clin. Nutr. 26: 998 (1973)

16. Ferris, S. and Himwich, H. E.: The effect of hypoglycemia and age on the glycogen content of the various parts of the feline central nervous system. Am. J. Physiol., 146: 389 (1946). 
17. Freinkel, N. E., Metzger, B. E., Nitzan, N. M., Hare, J. W., Shambaugh, G. E., Marshall, R. T., Surmaczynska, B. Z., and Nagel. T. C.: Accelerated starvation and mechanisms for the conservation of maternal nitrogen during pregnancy. Isr. J. Med. Sci., 8: 426 (1972)

18. Goodner, C. J., and Thompson, D. J.: Glucose metabolism in the fetus in utero: the effect of maternal fasting and glucose loading in the rat. Pediatr. Res., I: 443 (1967).

19. Gorell, J. M.. Dolkart, P. H., and Ferrendelli, J. A.: Regional levels of glucose, amino acids, high energy phosphates, and cyclic nucleotides in the central nervous system during hypoglycemic stupor and behavioral recovery. J. Neurochem., 27: 1043 (1976).

20. Kliegman, R. M., Miettinen, E. L., and Adam, P. A. J.: Fetal and neonatal responses to maternal canine starvation: circulating fuels and neonatal glucose production. Pediatr. Res., (in press).

21. Kohle, S. J., and Vannucci, R. C.: Glycogen metabolism in fetal and postnatal rat brain: influence of birth. J. Neurochem., 28: 441 (1977).

22. Kraus, H., Schlenker, S.. and Schwedesky, D.: Developmental changes of cerebral ketone body utilization in human infants. Hoppe-Seyler's Z. Physiol. Chem., 355: 164 (1974)

23. Lewis, L. D., Ljunggren, B., Norberg, K., and Siesjö, B. K.: Changes in carbohydrate substances, amino acids and ammonia in the brain during insulininduced hypoglycemia. J. Neurochem., 23: 659 (1974).

24. Lewis, L. D., Ljunggren, B., Ratcheson, R. A., and Siesjo, B. K.: Cerebral energy state in insulin-induced hypoglycemia related to blood glucose and to EEG. J. Neurochem., 23: 673 (1974).

25. Lowry, O. H. and Passonneau, J. V.: The relationships between substrates and enzymes of glycolysis in brain. J. Biol. Chem., 239: 31 (1964).

26. Lowry, O. and Passonneau, J.: A Flexible System of Enzymatic Analysis. (Academic Press, Inc., New York, 1972)

27. Mann, L. I., Duchin. S., Halverstram, J., Magtrantonio, J., Weiss, R., and

Copyright (C) 1981 International Pediatric Research Foundation, Inc

$0031-3998 / 81 / 1505-0859 \$ 02.00 / 0$
Schulman, J.: The effect of hypoglycemia on fetal brain function and metabolism. Am. J. Obstet. Gynecol., I17: 45 (1973).

28. Nelson, S. R. Schulz, D. W., Passonneau, J. V., and Lowry O. H.: Control of glycogen levels in brain. J. Neurochem, 15: 1217 (1968).

29. Newsholme, E. A.: Carbohydrate metabolism in vivo: regulation of the blood glucose level. Clin. Endocrinol. Metab.. 5: 543 (1976).

30. Norberg, K, and Siesjo, B. K. Oxidative metabolism of the cerebral cortex of the rat in severe insulin-induced hypoglycaemia. J. Neurochem., 26: 345 (1976).

31. Novak. M.: Colorimetric ultramicro method for the determination of free fatty acids. J. Lipid Res., 6: 431 (1965).

32. Owen, O. E., Morgan, A. P., Kemp, H. G., Sullivan, J. M., Herrerg, M. G., and Cahill, G. F.: Brain metabolism during fasting. J. Clin. Invest., 46: 1589 (1967).

33. Rolleston, F. S., and Newsholme, E. A.: Control of glycolysis in cerebral cortex slices. J., Biochem. 104: 524 (1967).

34. Siesjö, B. K.: Brain energy metabolism. (John Wiley \& Sons, Chichester, 1978).

35. Simmons, M. A., Meschia, G., Makowski, L.. and Battaglia. F. C.: Fetal metabolic response to maternal starvation. Pediatrs. Res., 8: 830 (1974).

36. Vannucci, R. C. and Duffy, T. E.: Influence of birth on carbohydrate and energy metabolism in rat brain. Am. J. Physiol., 226: 933 (1974).

37. Dr. Adam unfortunately expired prior to publication of this work

38. The authors would like to express their appreciation to George Campbell for his technical assistance and to Dr. Sherman D. Sachs of the Department of Veterinary Medicine of Case Western Reserve University for surgical and general care of the beagles.

39. Requests for reprints should be addressed to: Dr. R. M. Kliegman, Department of Pediatrics, Division of Pediatric Metabolism, Bell Greve W 721, Cleveland Metropolitan General Hospital, 3395 Scranton Road. Cleveland, OH 44109 (USA).

40. Received for publication June 23, 1980

41. Accepted for publication September 16, 1980. 